\section{Revista de CIENCIAS AMBIENTALES Tropical Journal of Environmental Sciences}

Revista de Ciencias Ambientales (Trop J Environ Sci) e-ISSN: $2215-3896$

(Julio-Diciembre, 2021) . Vol 55(2): 157-179

DOI: https://doi.org/10.15359/rca.55-2.8

Open Access: www.revistas.una.ac.cr/ambientales e-mail: revista.ambientales@una.ac.cr

Pinos-Morocho D., Morales-Matute O. y Durán-López M. E.

\title{
Suelos de páramo: Análisis de percepciones de los servicios ecosistémicos y valoración económica del contenido de carbono en la sierra sureste del Ecuador
}

\author{
Paramo soils: analysis of perceptions of ecosystem services and economic valuation \\ of carbon content in the southeastern sierra of Ecuador
}

\author{
Daniela Pinos-Morocho ${ }^{1}$, Oscar Morales-Matute ${ }^{2}$, María Elisa Durán-López $^{3}$
}

[Recibido: 27 de abril 2020, Aceptado: 7 de enero 2021, Corregido: 04 de febrero 2021, Publicado: 1 de julio 2021]

\section{Resumen}

[Introducción]: El suelo del páramo contiene grandes cantidades de carbono orgánico, gracias a las bajas temperaturas que reducen la descomposición de la materia orgánica que se acumula. Estos suelos prestan un servicio ecosistémico importante a nivel global, al regular el nivel de dióxido de carbono emitido a la atmósfera, actuando como sumideros. Adicionalmente, el análisis de la percepción ambiental de estos servicios ecosistémicos ayudará a su conservación. [Objetivo]: Evaluar la percepción que tienen los pobladores sobre los servicios ecosistémicos y determinar el valor económico del contenido de carbono, en los suelos del páramo cercano al Bosque Protector Cubilán, Ecuador. [Metodología]: Mediante un muestreo intencional y un análisis descriptivo se evaluó cómo los habitantes de 3 comunas perciben los servicios ecosistémicos del páramo. Además, para determinar el valor económico del carbono se plantearon 2 escenarios (suelos intervenidos y naturales) y se aplicó el método que obtiene el costo del daño evitado a nivel global, por la mitigación del cambio climático. [Resultados]: Los pobladores perciben las 4 categorías utilizadas para definir los servicios ecosistémicos con una jerarquía marcada. Por otro lado, los suelos intervenidos pierden, en promedio, un $26 \%$ de contenido de carbono en comparación con el contenido de sitios naturales que, traducido a un valor económico, representa una pérdida monetaria del $6 \%$ aproximadamente. [Conclusiones]: Los servicios ecosistémicos de este páramo se perciben adecuadamente en su zona de influencia. Continuar con actividades antropogénicas (ganadería y/o agricultura) contribuye a una pérdida de contenido de carbono y económica.

Palabras clave: Cambio climático; conservación; dióxido de carbono; percepción del entorno; suelo.

\section{Abstract}

[Introduction]: The soil of the paramo contains large amounts of organic carbon, due to the low temperatures which reduce the decomposition of the organic matter that accumulates. These soils provide an important ecosystem service worldwide, acting as sinks by regulating the level of carbon dioxide emitted into the atmosphere. Additionally, the analysis of the environmental perception of these ecosystem services will help to their conservation. [Objective]: Evaluate the perception that inhabitants have on the ecosystem services and determine the economic

1 Ingeniera ambiental, profesional independiente, Ecuador; pinos8674@gmail.com, https://orcid.org/0000-0001-6436-897X

2 Investigador, Programa para el Manejo del Agua y del Suelo (PROMAS), Universidad de Cuenca, Ecuador; oscar.morales@ucuenca.edu.ec, https://orcid.org/0000-0003-2109-7828

3 Docente e investigadora en la Facultad de Ciencias Químicas, Universidad de Cuenca, Ecuador; elisa.duranl@ucuenca.edu.ec, https://orcid. org/0000-0003-1642-656X

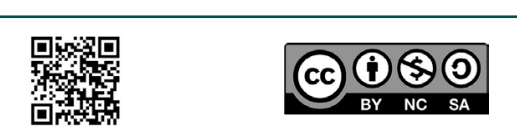

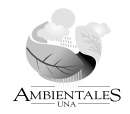




\section{Revista de CIENCIAS AMBIENTALES Tropical Journal of Environmental Sciences}

Revista de Ciencias Ambientales (Trop J Environ Sci) e-ISSN: 2215-3896

(Julio-Diciembre, 2021) . Vol 55(2): 157-179 DOI: https://doi.org/10.15359/rca.55-2.8 Open Access: www.revistas.una.ac.cr/ambientales e-mail: revista.ambientales@una.ac.cr

Pinos-Morocho D., Morales-Matute O. y Durán-López M. E.

value of the carbon content in the soils of the paramo near the Cubilán Protective Forest, Ecuador. [Methodology]: Through an intentional sampling and a descriptive analysis, the perception of the inhabitants of 3 communes on ecosystem services of the paramo was evaluated. In addition, to determine the economic value of carbon, 2 scenarios were proposed (intervened and natural soils) and the method that obtains the damage cost avoided globally due to the mitigation of climate change was applied. [Results]: The inhabitants perceive the 4 categories used to define the ecosystem services with a marked hierarchy. On the other hand, the intervened soils lose, on average, 26 $\%$ of carbon content compared to the content of natural sites, which, translated into an economic value, represents a monetary loss of approximately $6 \%$. [Conclusions]: The ecosystem services of this paramo are adequately perceived in its area of influence. Continuing with anthropogenic activities (livestock and/or agriculture) contributes to carbon content and economic losses.

Keywords: Carbon dioxide; climate change; conservation; perception of the environment; soil.

\section{Introducción}

Los páramos poseen una gran biodiversidad y brindan varios servicios ecosistémicos; por ejemplo, la regulación hídrica, captura de carbono, protección del suelo, conservación de la biodiversidad, entre otros (Calderón et al., 2013; Farley et al., 2011). No obstante, de los servicios mencionados, la captura y el contenido de carbono en el suelo es el que menos se conoce en Sudamérica (Ayala et al., 2014).

El suelo de páramo tiene la capacidad de acumular grandes cantidades de carbono, gracias a las bajas temperaturas que reducen las tasas de descomposición de la materia orgánica que se almacena en él (Castañeda \& Montes, 2017). Un estudio comparativo, realizado por la Organización de las Naciones Unidas para la Alimentación y la Agricultura (FAO, 2002), muestra la diferencia entre las cantidades de carbono que contienen distintos suelos: a) zonas áridas capturan $4 \mathrm{~kg} / \mathrm{m}^{2}$; b) regiones polares $21-24 \mathrm{~kg} / \mathrm{m}^{2}$; c) zonas tropicales $8-10 \mathrm{~kg} / \mathrm{m}^{2}$; d) páramos hasta $60 \mathrm{~kg} / \mathrm{m}^{2}$ (Ayala et al., 2014). De esta forma se pone en evidencia la función relevante que cumplen los suelos de páramo, pues al poseer tal potencial de captura de carbono ayudan en la mitigación de los efectos negativos ocasionados por el cambio climático (Castañeda \& Montes, 2017).

No obstante, un obstáculo para la conservación del páramo es la falta de conocimiento e interés de las comunidades aledañas e instituciones gubernamentales en los servicios ecosistémicos que brinda este ecosistema. Tal es el caso de ciertas zonas andinas de Venezuela, Colombia, Ecuador y Perú, en donde el páramo se encuentra infravalorado y sobreexplotado (Castañeda, 2016; Castro, 2011; Espinosa et al., 2014). Si bien existen estudios en los que se evalúan las percepciones que los habitantes tienen sobre los servicios ecosistémicos en países andinos, el énfasis se ha puesto sobre la disposición a pagar por ellos (Farley et al., 2011; Hayes et al., 2015, 2017; Higuera et al., 2013). De manera que es muy importante incluir las diferentes formas de valoración e interacción, más allá de una mirada económica, que tienen los habitantes del páramo con su entorno, puesto que las comunidades humanas influyen significativamente en las decisiones que se toman sobre el manejo y cuidado del ambiente (Caballero et al., 2017; Durán,

\begin{tabular}{|c|c|c|}
\hline 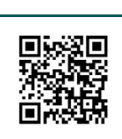 & (c) (i) () () () & 158 \\
\hline
\end{tabular}




\section{Revista de CIENCIAS AMBIENTALES Tropical Journal of Environmental Sciences}

Revista de Ciencias Ambientales (Trop J Environ Sci)

e-ISSN: 2215-3896

(Julio-Diciembre, 2021) . Vol 55(2): 157-179

DOI: https://doi.org/10.15359/rca.55-2.8

Open Access: www.revistas.una.ac.cr/ambientales e-mail: revista.ambientales@una.ac.cr

Pinos-Morocho D., Morales-Matute O. y Durán-López M. E.

2013). En Ecuador, dos tercios de la extensión total de páramos (1.5 millones ha) se encuentran fuera del Sistema Nacional de Áreas Protegidas (Farley et al., 2011).

De acuerdo con las consideraciones planteadas, el objetivo de este estudio es analizar la percepción ambiental que tienen los pobladores de 3 comunas aledañas al páramo, sobre los servicios ecosistémicos que obtienen de él y, determinar el valor económico del contenido de carbono orgánico en los suelos de esta zona al sur de Ecuador. Así, se vincula la participación social con un análisis técnico que respalde la conservación y el manejo adecuado de este ecosistema. Los resultados obtenidos pondrán en evidencia los diferentes servicios ecosistémicos que brinda el páramo ubicado al sureste de Ecuador, como un importante sumidero de carbono y como un generador de sentimientos de pertenencia para los habitantes de las comunas cercanas. Además, la información generada permitirá que los tomadores de decisiones encaminen acciones para conservar el páramo e incluirlo dentro del catastro de áreas protegidas.

\section{Marco teórico}

\subsection{Páramo}

Se considera al páramo como un ecosistema húmedo tropical ubicado normalmente a partir del límite forestal superior (Castañeda, 2016; Hofstede et al., 2014), dominado por vegetación herbácea y arbustiva (Crespo et al., 2009) y con paisajes bioclimáticos fríos (sobre los $3200 \mathrm{~m}$ s.n.m.) (Castañeda, 2016). El páramo no solo es un ecosistema de alta montaña tropical, sino también es un espacio de producción y trabajo con gran historia, cultura y política (Hofstede et al., 2014; Serrano \& Galárraga, 2015).

En Sudamérica, el páramo cubre aproximadamente $46000 \mathrm{~km}^{2}$ que se distribuyen entre Venezuela (5.2 \%), Colombia (30.5\%), Ecuador (39.8 \%) y Perú (24.6\%); formando parte importante de su biodiversidad (Serrano \& Galárraga, 2015). En Ecuador, el páramo ocupa alrededor de 1500000 ha, convirtiéndose así en el país que tiene la mayor superficie de páramo con respecto a su extensión total (Mena \& Medina, 2002).

En cuanto a la biodiversidad de los páramos, esta se define mejor por ser única antes que por tener riqueza, pues cuenta con un alto endemismo y especialización, tanto a nivel genético como de paisajes, promovidos por su ocurrencia aislada y fragmentada (Castro, 2011). Existen dos clasificaciones con respecto a este ecosistema: el páramo natural, sin cambios en el uso del suelo por intervención humana y que originalmente está cubierto por pajonal con bosquetes y algunos pantanos (Castañeda, 2016); y por otra parte, el páramo intervenido que alberga poblaciones humanas con organizaciones culturales que aportan al enriquecimiento patrimonial del planeta, y está considerado también como un espacio social que genera beneficios económicos (Serrano \& Galárraga, 2015).

\begin{tabular}{|c|c|c|c|c|c|}
\hline 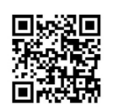 & (c) (1) () (2) & $\overbrace{\text { AMBENEMIIISS }}$ & $\frac{O \%}{20}$ & 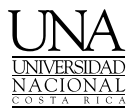 & 159 \\
\hline
\end{tabular}




\section{Revista de CIENCIAS AMBIENTALES Tropical Journal of Environmental Sciences}

Revista de Ciencias Ambientales (Trop J Environ Sci) e-ISSN: 2215-3896

(Julio-Diciembre, 2021) . Vol 55(2): 157-179

DOI: https://doi.org/10.15359/rca.55-2.8

Open Access: www.revistas.una.ac.cr/ambientales e-mail: revista.ambientales@una.ac.cr

Pinos-Morocho D., Morales-Matute O. y Durán-López M. E.

\subsection{Servicios ecosistémicos}

Los servicios ecosistémicos se definen como los beneficios que las poblaciones humanas obtienen de las funciones de los ecosistemas (Millennium Ecosystem Assessment, 2005), ya sea de manera directa o indirecta (Camacho \& Ruiz, 2012). La variedad de servicios proporcionados por los ecosistemas puede clasificarse en diferentes categorías, para simplificar la forma en la que los valoramos e identificamos (Camacho \& Ruiz, 2012; IPCC, 2014). Probablemente una de las clasificaciones más difundidas y aceptadas a nivel mundial es la que ofrece la Evaluación de los Ecosistemas del Milenio (2005), que se basa en 4 lineamientos funcionales, los cuales se encuentran descritos en el Cuadro 1.

Cuadro 1. Clasificación de servicios ecosistémicos y sus funciones.

Table 1. Classification of ecosystem services and their functions.

\begin{tabular}{ll}
\hline \multicolumn{1}{c}{ Clasificación } & \multicolumn{1}{c}{ Función } \\
\hline $\begin{array}{l}\text { Servicios ecosistémicos de } \\
\text { aprovisionamiento (SEA) }\end{array}$ & $\begin{array}{l}\text { Consideran los productos obtenidos de los ecosistemas, como alimentos, agua } \\
\text { dulce para consumo, leña, recursos genéticos, entre otros. }\end{array}$ \\
Servicios ecosistémicos de regulación (SER) & $\begin{array}{l}\text { Son aquellos beneficios que se obtienen de la regulación de los diferentes } \\
\text { procesos ecosistémicos, como la regulación del clima, captura, contenido y } \\
\text { almacenamiento de carbono, aire limpio, regulación y saneamiento de agua, } \\
\text { polinización, entre otros. }\end{array}$ \\
Servicios ecosistémicos culturales (SEC) & $\begin{array}{l}\text { Son beneficios no materiales que proveen los ecosistemas, como el bienestar } \\
\text { espiritual y religioso, la recreación y el turismo, la estética paisajística, la } \\
\text { identidad de un sitio, la herencia cultural, entre otros. }\end{array}$ \\
Cervicios ecosistémicos de soporte (SES) & $\begin{array}{l}\text { Catalogados como uno de los servicios más difíciles de identificar, son necesarios } \\
\text { para la producción de otros servicios ecosistémicos, como la formación de suelos, } \\
\text { el reciclaje de nutrientes, la producción primaria, entre otros. }\end{array}$ \\
\hline
\end{tabular}

En los páramos existen servicios ecosistémicos de gran importancia a nivel biológico, hidrológico, social, cultural y económico (Ayala et al., 2014). En este ecosistema, el servicio de regulación de captura y contenido de carbono se obtiene cuando la materia orgánica permanece aislada del oxígeno y se forman los sumideros o depósitos de carbono (Castañeda \& Montes, 2017). Los suelos del mundo contienen más carbono que la vegetación y la atmósfera juntas (Swift, 2001), y el suelo de páramo es considerado como uno de los mayores reservorios terrestres de carbono orgánico (Ayala et al., 2014). La concentración de carbono en el suelo aumenta cuando existe vegetación natural (briofitas y angiospermas arbustivas), pues esta aporta una gran cantidad de materia orgánica (Castañeda, 2016). Asimismo, aísla y protege el suelo del golpe de la precipitación que se acumula, minimiza la escorrentía y evita el rompimiento de agregados superficiales en partículas de tamaño transportable (Yang et al., 2014). La vegetación también protege el suelo de la incidencia de radiación solar, minimizando la descomposición de la materia orgánica.

En contraposición, el aprovechamiento de los servicios ecosistémicos culturales que proveen los páramos (bienestar espiritual, recreación, estética paisajística, identidad y herencia cultural, entre otros) atrae la presencia de los seres humanos; arriesgando el potencial del contenido de

\begin{tabular}{|c|c|c|c|c|c|}
\hline 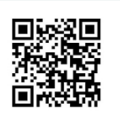 & (c) (1) (9) & 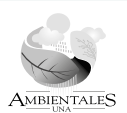 & $\frac{O \%}{2)}$ & 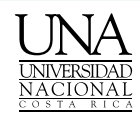 & 160 \\
\hline
\end{tabular}




\section{Revista de CIENCIAS AMBIENTALES Tropical Journal of Environmental Sciences}

Revista de Ciencias Ambientales (Trop J Environ Sci) e-ISSN: 2215-3896

(Julio-Diciembre, 2021) . Vol 55(2): 157-179

DOI: https://doi.org/10.15359/rca.55-2.8

Open Access: www.revistas.una.ac.cr/ambientales e-mail: revista.ambientales@una.ac.cr

Pinos-Morocho D., Morales-Matute O. y Durán-López M. E.

carbono en los suelos cuando no existe un manejo adecuado que conserve sus funciones (Serrano \& Galárraga, 2015).

Bajo esta premisa nace la iniciativa de emplear mecanismos de mercado, con la finalidad de proteger y conservar la naturaleza; entre estos, se encuentra el mercado de carbono. Este ha sido planteado como una de las soluciones a la crisis climática y ambiental que enfrenta el planeta, a causa de las emisiones de gases de efecto invernadero. Dichas emisiones se incrementan por las alteraciones en la cobertura y el uso del suelo debido a actividades productivas (cultivos y ganadería) (Moreano, 2012).

\subsection{Percepción ambiental}

La percepción humana es un proceso cambiante, pues en ella influyen diferentes circunstancias sociales que intervienen en la realidad, como la cultura de pertenencia, el grupo y la clase social (Durán, 2013). La percepción ambiental es concebida como la forma en que un individuo entiende y valorara su entorno, a través de sus vivencias y experiencias; a su vez, esta influye significativamente en las decisiones que toma el ser humano sobre su ambiente (Fernández, 2008).

En consecuencia, una persona toma conciencia y comprende, desde su punto de vista, un sentido amplio de la realidad para construir y transmitir los modelos culturales e ideológicos (Durand, 2008). Adicionalmente, la cultura y procedencia del individuo inciden en el desarrollo de la percepción ambiental de los adultos (Durán et al., 2016).

Dada la actual crisis ambiental, es fundamental estudiar la dimensión socioambiental, puesto que los seres humanos modificamos los ecosistemas en función de nuestras percepciones e intereses (Caballero-Serrano et al. 2017). Estas investigaciones posibilitan que se visualice la importancia que las personas asignan, de forma individual y grupal, a los ecosistemas en donde habitan y permiten dirigir esfuerzos para la conservación ambiental.

\section{Metodología}

\subsection{Descripción del área}

El estudio se realizó en una zona de páramo ubicada al norte del Bosque Protector Cubilán, al sureste de Ecuador en la provincia de Cañar (Figura 1); sin embargo, no se encuentra dentro de los límites del área de conservación. Abarca una superficie de 757.83 ha y se ubica entre 3260 y 3500 m s.n.m. La temperatura media anual en esta cordillera va de 8.32 a $8.8^{\circ} \mathrm{C}(\mathrm{Ca}-$ macho, 2013; Córdova et al., 2016); el clima es de tipo frío a muy frío y la precipitación anual es 1177.9 mm (Fundación ECOHOMODE, 2006). La comunidad vegetal más abundante en este páramo es el pajonal, cuya especie representativa es Stipa ichu (Poaceae), conocida como paja brava o paja ichu, con hojas lineales, delgadas, angulosas, que crecen formando manojos (Barreno, 2013; Hofstede et al., 2014). Íñiguez et al. (2016) indican que los suelos de estos páramos

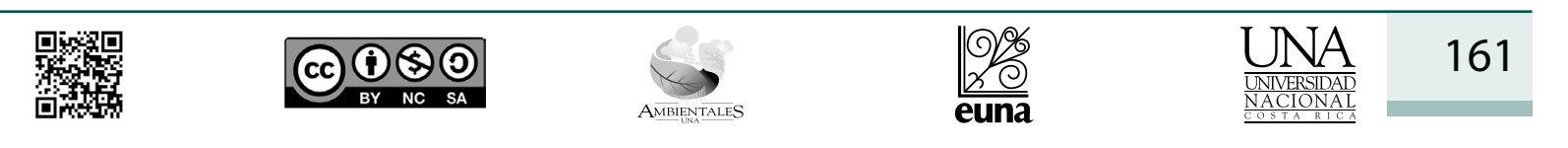




\section{Revista de CIENCIAS AMBIENTALES Tropical Journal of Environmental Sciences}

Revista de Ciencias Ambientales (Trop J Environ Sci) e-ISSN: 2215-3896

(Julio-Diciembre, 2021) . Vol 55(2): 157-179

DOI: https://doi.org/10.15359/rca.55-2.8

Open Access: www.revistas.una.ac.cr/ambientales e-mail: revista.ambientales@una.ac.cr

Pinos-Morocho D., Morales-Matute O. y Durán-López M. E.

principalmente corresponden a dos grupos: Andosoles e Histosoles, en función a la clasificación del World Reference Base for Soil Resources.

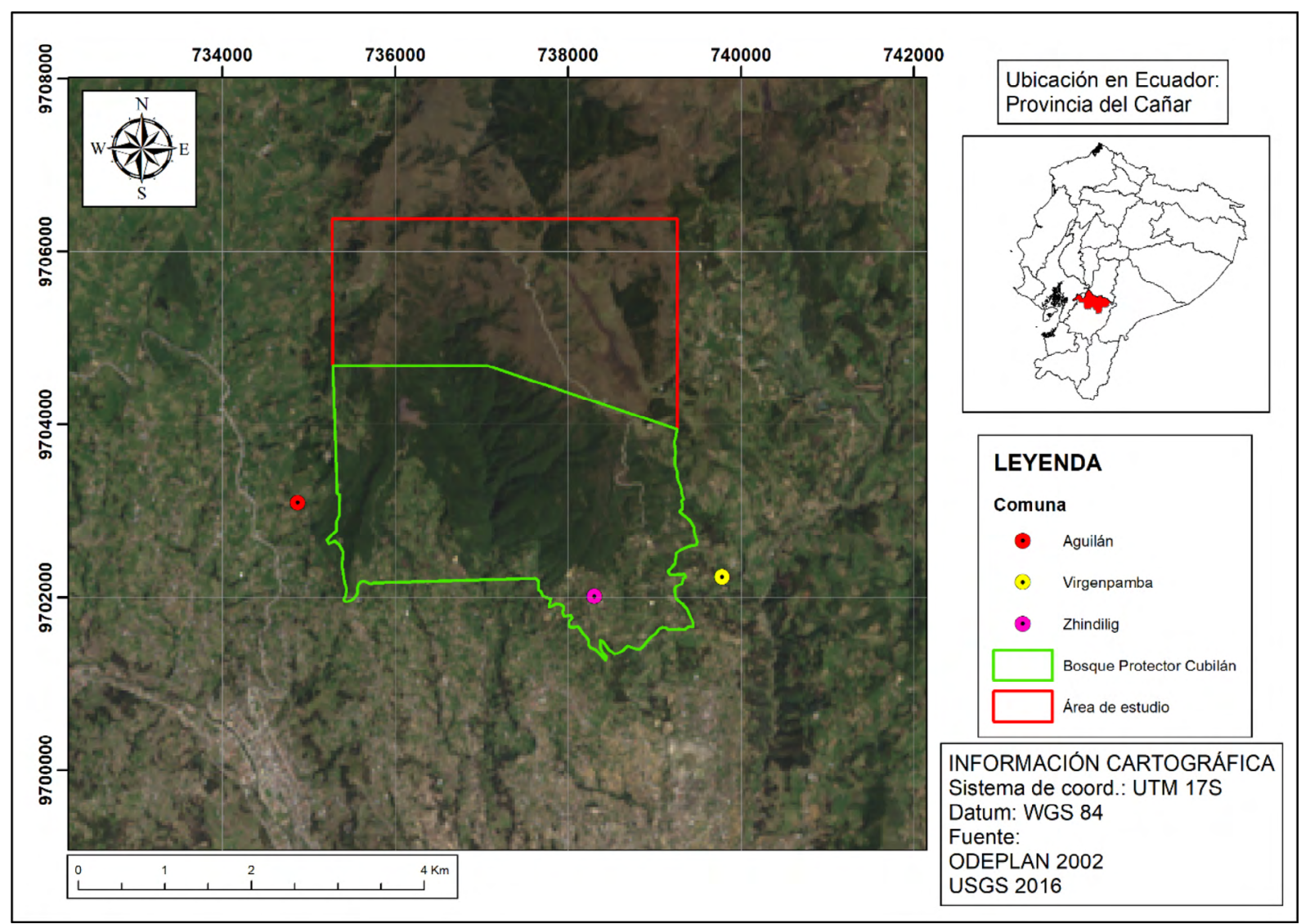

Figura 1. Mapa referencial del área de estudio.

Figure 1. Reference map of the study area.

Dado que uno de los objetivos fue establecer la percepción ambiental que tienen los habitantes de zonas cercanas al sitio de estudio, se seleccionaron las comunas de Aguilán, Virgenpamba y Zhindilig, todas ellas ubicadas en un radio de aproximadamente $5 \mathrm{~km}$ con relación al área de estudio (Figura 1).

\subsection{Evaluación de la percepción ambiental sobre servicios ecosistémicos}

El grupo meta (en las 3 comunas) fue seleccionado mediante un muestreo intencional (Otzen \& Manterola, 2017; Shepardson et al., 2009) e incluyó 66 individuos (33 hombres y 33 mujeres) entre 40 y 80 años, dado que las personas en este rango etario conocen mejor el entorno en el que se han desarrollado y pueden distinguir los cambios en su ambiente. La participación fue voluntaria.

\begin{tabular}{|c|c|c|}
\hline 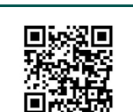 & (c) (i) (5) (0) & 162 \\
\hline
\end{tabular}




\section{Revista de CIENCIAS AMBIENTALES Tropical Journal of Environmental Sciences}

Cuadro 2. Categorías contempladas dentro de la encuesta. Table 2. Categories included in the survey.

\begin{tabular}{lc}
\hline \multicolumn{1}{c}{ Categoría } & No. de preguntas \\
\hline Servicios ecosistémicos de & 4 \\
aprovisionamiento (SEA) & \\
Servicios ecosistémicos de regulación (SER) & 5 \\
Servicios ecosistémicos culturales (SEC) & 6 \\
Servicios ecosistémicos de soporte (SES) & 4 \\
\hline
\end{tabular}

${ }^{\star}$ Número total de preguntas $=10$

guntas, con la finalidad de evidenciar el contacto directo que tienen las poblaciones con el páramo (Cuadro 2).

La información obtenida se analizó de forma descriptiva, al agrupar conceptos y patrones de respuestas de los comuneros, en 4 categorías de percepción sobre el páramo y los servicios ecosistémicos asociados (Cuadro 3).

Cuadro 3. Categorías para analizar la percepción ambiental sobre los servicios ecosistémicos del páramo. Table 3. Categories to analyze the environmental perception on the ecosystem services of the paramo.

\begin{tabular}{lll}
\hline \multicolumn{1}{c}{ Categoría ${ }^{*}$} & \multicolumn{1}{c}{ Concepto } & \multicolumn{1}{c}{ Elementos percibidos } \\
\hline $\begin{array}{l}\text { Servicios ecosistémicos de } \\
\text { aprovisionamiento (SEA) } \\
\text { Servicios ecosistémicos de regulación (SER) }\end{array}$ & $\begin{array}{l}\text { Bienes materiales que sustentan las } \\
\text { actividades del grupo meta } \\
\text { Beneficios que influyen sobre la calidad alimentos, plantas } \\
\text { de vida del grupo meta } \\
\text { Servicios ecosistémicos culturales (SEC) } \\
\text { Contacto directo e indirecto que tiene } \\
\text { el grupo meta con el ecosistema }\end{array}$ & $\begin{array}{l}\text { Agua, leña, } \\
\text { medicinales. } \\
\text { Aire limpio, regulación y } \\
\text { saneamiento del agua. } \\
\text { Belleza escénica, fauna y flora } \\
\text { hermosas, tranquilidad, identidad } \\
\text { de sitio, patrimonio. }\end{array}$ \\
& $\begin{array}{l}\text { Funciones que favorecen el desarrollo } \\
\text { de los procesos ecológicos y biológicos }\end{array}$ & $\begin{array}{l}\text { Formación de suelos, reciclaje de } \\
\text { nutrientes, fotosíntesis. }\end{array}$ \\
\hline
\end{tabular}

${ }^{\star}$ Basada en la Evaluación de los Ecosistemas del Milenio (2005).

\subsection{Ubicación de los puntos de muestreo de suelo}

La ubicación de los sitios para recolectar las muestras se realizó de manera fundamentada, con base en las recomendaciones del Instituto Nacional de Investigaciones Agropecuarias del Ecuador (INIAP, 2016). Uno de los principales criterios de selección del sitio es la accesibilidad, la cual permite la inclusión o exclusión de ciertas áreas. Otro criterio considerado es la distribución casi homogénea de los suelos, que se consigue principalmente al analizar mapas de tipos de suelo disponibles. No obstante, es imprescindible la inspección de campo, porque de acuerdo con la vegetación presente en un área determinada, es posible estimar la presencia de suelos con características similares.

Como parte de la metodología aplicada, otra de las unidades de categorización considerada fue la pendiente del terreno, debido a que se observó que este parámetro influye directamente

\begin{tabular}{|c|c|c|c|c|c|}
\hline 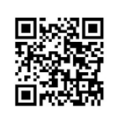 & (c) (1) (9) & $\underset{\text { AMBENTALES }}{\leftrightarrow}$ & $\frac{9 \%}{2 \%}$ & $\frac{\text { UNA }}{\frac{\text { UNIIERSIDAD }}{\text { NASIONAL }}}$ & 163 \\
\hline
\end{tabular}




\section{Revista de CIENCIAS AMBIENTALES Tropical Journal of Environmental Sciences}

Revista de Ciencias Ambientales (Trop J Environ Sci) e-ISSN: 2215-3896

(Julio-Diciembre, 2021) . Vol 55(2): 157-179 DOI: https://doi.org/10.15359/rca.55-2.8 Open Access: www.revistas.una.ac.cr/ambientales e-mail: revista.ambientales@una.ac.cr

Pinos-Morocho D., Morales-Matute O. y Durán-López M. E.

sobre las características del suelo, como su cobertura vegetal y los usos actuales y futuros (Villar et al., 2013). Para elaborar el mapa de pendientes se utilizó información cartográfica base (modelo de elevación digital del terreno), recopilada de la página del Sistema Nacional de Información del Ecuador, disponible en escala 1:50 000. El mapa de pendientes fue generado según los 7 rangos (desde "Plana" hasta "Muy fuerte") establecidos por la clasificación del Ministerio de Agricultura, Ganadería, Acuacultura y Pesca (MAGAP et al., 2010).

Una vez ubicados los 7 puntos de forma preliminar, se realizó un recorrido en la zona de estudio para definir exactamente los 7 lugares definitivos de muestreo, con la ayuda de un equipo de posicionamiento global (GPS). Estos puntos se ubicaron en un rango altitudinal entre los 3 360 y 3450 m s.n.m. (Cuadro 4 y Figura 2).

Cuadro 4. Características de los puntos de muestreo.

Table 4. Characteristics of the sampling points.

\begin{tabular}{lcccc}
\hline Clasificación de pendientes [\%] (MAGAP, 2010) & Puntos de muestreo $^{*}$ & X & Y & Altitud $^{\text {Pana (0-2) }}$ \\
\hline Plana & Punto 1 & 738743 & 9704388 & 3382 \\
Muy suave (2-5) & Punto 2 & 738312 & 9704872 & 3385 \\
Suave (5-12) & Punto 3 & 736609 & 9705911 & 3360 \\
Media (12-25) & Punto 4 & 738078 & 9705204 & 3393 \\
Media a fuerte (25-40) & Punto 5 & 737175 & 9705493 & 3490 \\
Fuerte (40-70) & Punto 6 & 736158 & 9705668 & 3418 \\
Muy fuerte (>70) & Punto 7 & 735486 & 9705986 & 3450
\end{tabular}

${ }^{*}$ Los sitios de muestreo se encuentran referidos de acuerdo con el mapa mostrado en la Figura 2.

\begin{tabular}{|c|c|c|}
\hline 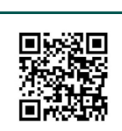 & (c) (1) () (2) & 164 \\
\hline
\end{tabular}




\section{Revista de CIENCIAS AMBIENTALES Tropical Journal of Environmental Sciences}

Revista de Ciencias Ambientales (Trop J Environ Sci) e-ISSN: 2215-3896

(Julio-Diciembre, 2021) . Vol 55(2): 157-179 DOI: https://doi.org/10.15359/rca.55-2.8 Open Access: www.revistas.una.ac.cr/ambientales e-mail: revista.ambientales@una.ac.cr

Pinos-Morocho D., Morales-Matute O. y Durán-López M. E.

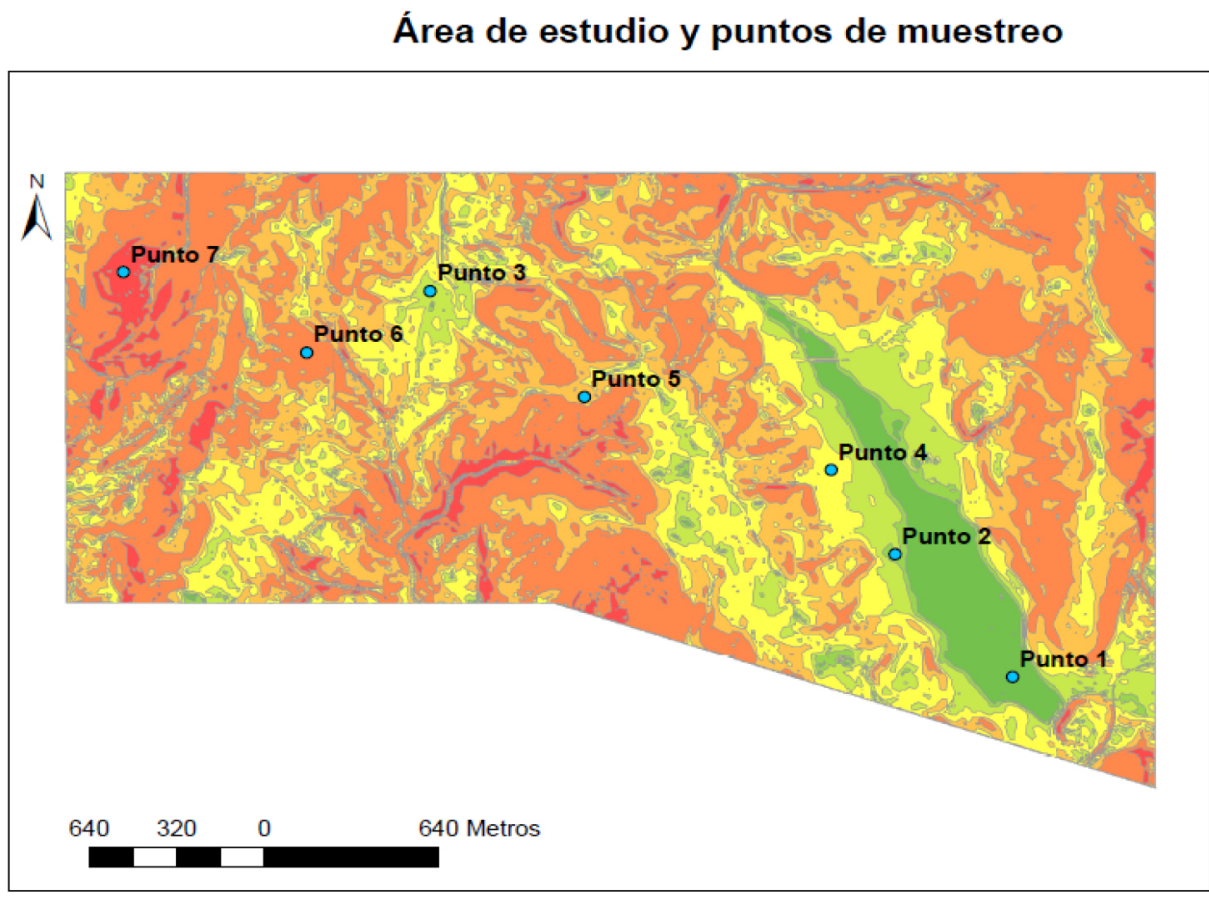

Identificación

- Puntos de muestreo

Pendientes [\%]

Rangos

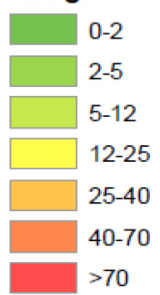

$>70$

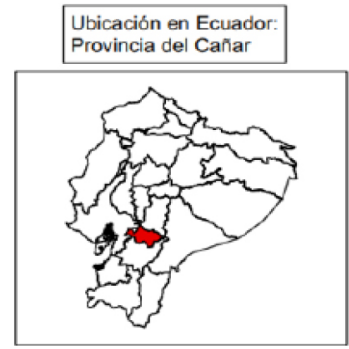

INFORMACIÓN CARTOGRÁFICA

Sistema de coord.: UTM $17 \mathrm{~S}$

Datum: WGS 84

Fuente: USGS 2016

Figura 2. Mapa de pendientes del área de estudio y puntos de muestreo.

Figure 2. Slope map of the study area and sampling points.

\subsection{Diferenciación de puntos intervenidos y naturales}

Para establecer si los suelos de ecosistemas conservados albergan mayores cantidades de carbono que los sitios intervenidos, se plantearon dos escenarios de muestreo: a) El primero indica el valor económico del carbono en sitios de páramo natural, que está cubierto por comunidades vegetales de almohadilla (estratos menores a $10 \mathrm{~cm}$ ), herbácea y arbustiva (estratos entre 20 y $150 \mathrm{~cm}$ ) con bosquetes y algunos pantanos, sin cambios en el uso del suelo original. b) El segundo indica el valor del carbono en sitios de páramo intervenido, donde se realizaban actividades de agricultura y ganadería desde una fecha desconocida hasta aproximadamente el año 2006, en donde cesan por aplicación de un plan de manejo ambiental. Sin embargo, la intervención fue tal que se cambió por completo la cobertura del suelo, llevándola a una transición desde pajonal hasta pastos para ganadería. Para procurar diferenciar los dos escenarios, se clasificó cada uno de los siete sitios mediante la observación en campo.

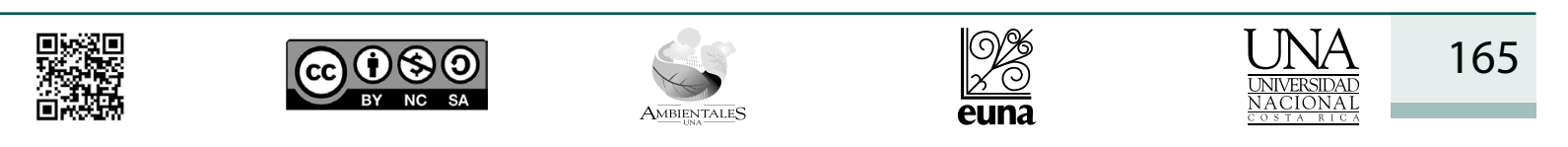




\section{Revista de CIENCIAS AMBIENTALES Tropical Journal of Environmental Sciences}

Revista de Ciencias Ambientales (Trop J Environ Sci) e-ISSN: 2215-3896

(Julio-Diciembre, 2021) . Vol 55(2): 157-179 DOI: https://doi.org/10.15359/rca.55-2.8 Open Access: www.revistas.una.ac.cr/ambientales e-mail: revista.ambientales@una.ac.cr

Pinos-Morocho D., Morales-Matute O. y Durán-López M. E.

Con base en la observación in situ, se definieron los puntos 2, 5, 6 y 7 como sitios naturales, por conservar las características típicas del páramo; mientras que los puntos 1,3 y 4 se identificaron como sitios intervenidos, puesto que evidenciaban acción antropogénica por pastoreo de ganado bovino y equino.

\subsection{Muestreo de suelos para determinar el contenido de carbono orgánico}

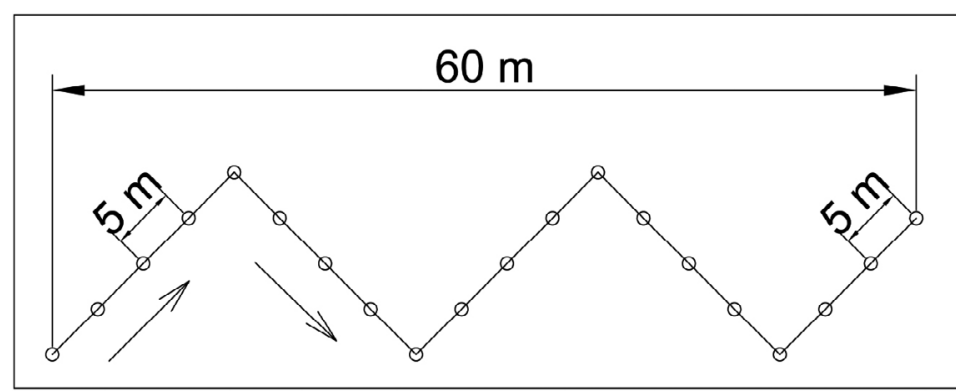

Figura 3. Ejemplo del recorrido en zigzag para la toma de submuestras en un punto.

Figure 3. Example of the zig-zag path for taking subsamples at a point.

Para estimar el contenido de carbono orgánico se realizó el muestreo en el mes de abril de 2018 y se siguió el procedimiento establecido por el Instituto Nacional de Investigaciones Agropecuarias (INIAP), que consistió en colectar muestras compuestas juntando 20 submuestras de suelo por cada punto de muestreo. Las submuestras se recogieron efectuando un recorrido en zigzag, a una distancia aproximada de $5 \mathrm{~m}$, sobre el área de cada punto de muestreo y a una profundidad de $20 \mathrm{~cm}$ (Figura 3). Estas muestras compuestas fueron analizadas en el Laboratorio de Análisis de Suelos de la Facultad de Ciencias Químicas de la Universidad de Cuenca, usando el método de Walkley-Black, cuyo principio es la oxidación incompleta del carbono orgánico y comprende una combustión húmeda de la materia orgánica, con una mezcla de dicromato de potasio $\left(\mathrm{K}_{2} \mathrm{Cr}_{2} \mathrm{O}_{7}\right)$ y ácido sulfúrico $\left(\mathrm{H}_{2} \mathrm{SO}_{4}\right)$. Cada muestra fue secada al ambiente y pasó por un tamiz ASTM No 40 (abertura $=0.425 \mathrm{~mm}$ ) (Guamán, 2018).

Adicionalmente, se tomaron muestras con anillos de Kopecky $\left(100 \mathrm{~cm}^{3}\right)$ para definir la densidad aparente $\left[\mathrm{g} / \mathrm{cm}^{3}\right.$ ] (Yang et al., 2014), siguiendo el procedimiento propuesto por el Laboratorio de Análisis de Suelos del Programa para el Manejo del Agua y el Suelo (PROMAS) de la Universidad de Cuenca. La profundidad de este muestreo también fue de $20 \mathrm{~cm}$ y se recolectaron 3 muestras inalteradas por cada sitio de monitoreo.

Con los resultados de contenido de carbono orgánico y la densidad aparente de las 7 muestras de suelo, se extrapoló a la medida estándar de reporte de contenido de carbono (toneladas de $\mathrm{CO}_{2}$ por hectárea), para determinar el contenido actual (Ecuación 1), de acuerdo con lo establecido por Castro (2011).

$$
\text { Contenido C suelo }\left[\frac{\operatorname{ton} \mathrm{Co}_{2}}{h a}\right]=(\rho * h * C * 100) * \frac{44}{12}(\mathbf{E} . \mathbf{1})
$$

Dónde:

$\rho=$ Densidad del suelo $\left[\mathrm{g} / \mathrm{cm}^{3}\right]$

$h=$ Profundidad a la que se toma la muestra $[\mathrm{cm}]$

$C=$ Porcentaje en peso de carbono orgánico en el suelo

\begin{tabular}{|c|c|c|}
\hline 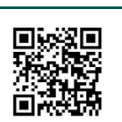 & (c) (1) () (2) & 166 \\
\hline
\end{tabular}




\section{Revista de CIENCIAS AMBIENTALES Tropical Journal of Environmental Sciences}

Revista de Ciencias Ambientales (Trop J Environ Sci) e-ISSN: 2215-3896

(Julio-Diciembre, 2021) . Vol 55(2): 157-179

DOI: https://doi.org/10.15359/rca.55-2.8

Open Access: www.revistas.una.ac.cr/ambientales e-mail: revista.ambientales@una.ac.cr

Pinos-Morocho D., Morales-Matute O. y Durán-López M. E.

\subsection{Costo social del carbono}

Para la valoración económica del contenido de carbono, se utilizó el método del costo del daño evitado a nivel global por la mitigación del cambio climático (Castro, 2011). Se proyectaron 4 escenarios cronológicos, tomando los precios del carbono emitidos en distintos informes realizados a nivel mundial, para los años 2011, 2017, 2020 y 2030 (Cuadro 5). Para el escenario correspondiente al primer año se tomó la cifra reportada por Castro (2011), que indica un valor referencial para una comparación relativa a la variación del costo del carbono en función del tiempo. Para el segundo escenario (año 2017) se empleó el dato proporcionado por el Banco Mundial en su informe "Fijación del precio del carbono" (2017), siendo el valor más cercano reportado hasta la fecha en que se realizó este trabajo. Los precios previstos para el tercer y cuarto escenario (año 2020 y 2030) fueron generados por el Banco Mundial en su informe State and Trends of Carbon Pricing, donde se indica que los costos que varían entre rangos (40-80 y 50-100 USD/ton $\mathrm{CO}_{2}$ ) son coherentes con el objetivo de temperatura del Acuerdo de París (World Bank et al., 2017).

\subsection{Valoración económica del contenido de carbono en el suelo}

El contenido de carbono en ton $\mathrm{CO}_{2} /$ ha (Ecuación 1) se multiplicó por cada precio establecido en el Cuadro 5. El resultado de la Ecuación 2 se extrapoló a toda la superficie del área de estudio (757.83 ha); de esa manera se obtuvo el valor económico total del contenido de carbono para los diferentes años.

$$
V \text { carbono }\left[\frac{U S D}{h a}\right]=\text { Contenido } C \text { suelo }\left[\frac{\operatorname{ton} \mathrm{CO}_{2}}{h a}\right] * \text { Costo social del } C\left[\frac{\mathrm{USD}}{\text { ton } \mathrm{CO}_{2}}\right](\mathrm{E}
$$

Para determinar la pérdida monetaria generada por el cambio del contenido de carbono en un suelo de páramo natural versus un suelo intervenido, se compararon entre sí los valores económicos calculados para cada uno de estos dos escenarios.

\section{Resultados}

\subsection{Evaluación de la percepción ambiental sobre los servicios ecosistémicos que brinda el páramo}

En cada una de las comunas se identificaron las 4 categorías definidas para los servicios ecosistémicos. Como se observa en el Cuadro 6, la relación establecida entre el páramo y sus

\begin{tabular}{|c|c|c|c|c|c|}
\hline 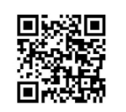 & (c) (i) () & $\underset{\text { AMBIENTALS }}{\leftrightarrow}$ & $\frac{1 \%}{2 \%}$ & 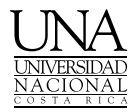 & 167 \\
\hline
\end{tabular}




\section{Revista de CIENCIAS AMBIENTALES Tropical Journal of Environmental Sciences}

Revista de Ciencias Ambientales (Trop J Environ Sci) e-ISSN: 2215-3896

(Julio-Diciembre, 2021) . Vol 55(2): 157-179

DOI: https://doi.org/10.15359/rca.55-2.8

Open Access: www.revistas.una.ac.cr/ambientales e-mail: revista.ambientales@una.ac.cr

Pinos-Morocho D., Morales-Matute O. y Durán-López M. E.

servicios ecosistémicos fue la siguiente: a) agua como principal elemento que este ecosistema les brinda (SEA); b) vegetación en buen estado y aire limpio que influyen en su salud (SER); c) sentimiento de pertenencia y cercanía a este ecosistema que contribuye en el bienestar y calidad de vida de los habitantes (SEC); y d) la importancia de la conservación del páramo para garantizar el mantenimiento de los procesos biológicos y ecológicos, principalmente en el suelo (SES). Aun cuando la principal actividad económica de las 3 localidades es la agricultura y ganadería, estos resultados evidencian el alto grado de comprensión que tienen los comuneros sobre los servicios ecosistémicos del páramo y la importancia de estos como agentes de su calidad de vida.

Cuadro 6. Información que argumenta la categorización de servicios ecosistémicos utilizada.

Table 6. Information that supports the categorization of ecosystem services used.

\begin{tabular}{lll}
\hline \multicolumn{1}{c}{ Categorías de servicios ecosistémicos } & \multicolumn{1}{c}{ Elementos percibidos } & \multicolumn{1}{c}{ Cita textual representativa } \\
\hline $\begin{array}{l}\text { Servicios ecosistémicos de } \\
\text { aprovisionamiento (SEA) } \\
\text { Servicios ecosistémicos de regulación (SER) }\end{array}$ & $\begin{array}{l}\text { Agua, leña, paja, alimentos, plantas } \\
\text { medicinales. } \\
\text { Aire limpio, regulación y } \\
\text { saneamiento del agua. }\end{array}$ & $\begin{array}{l}\text { "El agüita que viene del páramo nunca } \\
\text { falta" -Comunera de Aguilán. } \\
\text { "Siento que el aire del páramo es más } \\
\text { fresco y limpio, me siento más sano" } \\
\text { Servicios ecosistémicos culturales (SEC) }\end{array}$ \\
$\begin{array}{l}\text { Belleza escénica, fauna y flora } \\
\text { hermosas, tranquilidad, identidad } \\
\text { de sitio, patrimonio. } \\
\text { Servicios ecosistémicos de soporte (SES) } \\
\text { Formación de suelos, reciclaje de } \\
\text { nutrientes, fotosíntesis. }\end{array}$ & $\begin{array}{l}\text { "Yo me siento cómoda en el páramo } \\
\text { a diferencia de estar en la ciudad" - } \\
\text { Comunera de Zhindilig. } \\
\text { "El suelo se nutre solo, y sabemos que } \\
\text { es un proceso continuo" -Comunera } \\
\text { de Virgenpamba. }\end{array}$ \\
\hline
\end{tabular}

Como se esperaba, los servicios ecosistémicos mayormente percibidos fueron los de abastecimiento (SEA) y culturales (SEC) (> $50 \%$ de encuestados); mientras que el tercer y cuarto lugar fueron para los servicios de regulación (SER > $40 \%$ ) y soporte (SES 30 $\%$ aprox.), puesto que son los más difíciles de evidenciar. De forma general en las 3 comunas, la percepción más baja fue para los SES por ser una categoría de difícil reconocimiento. Por el contrario, los SEC tuvieron un nivel alto de apreciación, lo que demuestra que los comuneros tienen un sentido de pertenencia con el páramo y que lo reconocen como parte de su identidad (Figura 4).

La comuna de Aguilán demostró ser aquella con la percepción más elevada para los SEA y SEC (59

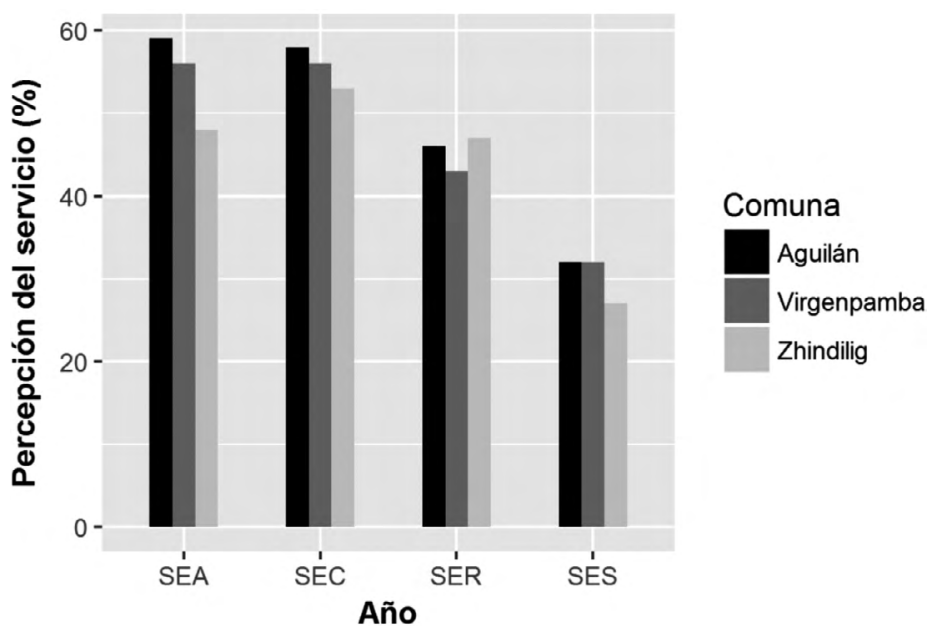

Figura 4. Percepción de servicios ecosistémicos en tres comunas. Figure 4. Perception of ecosystem services in three communes.

\begin{tabular}{|c|c|c|}
\hline 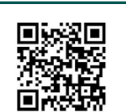 & (c) $\underset{\mathrm{BY}}{(\mathrm{i})}(\mathrm{NC}$ & 168 \\
\hline
\end{tabular}




\section{Revista de CIENCIAS AMBIENTALES Tropical Journal of Environmental Sciences}

Revista de Ciencias Ambientales (Trop J Environ Sci) e-ISSN: 2215-3896

(Julio-Diciembre, 2021) . Vol 55(2): 157-179

DOI: https://doi.org/10.15359/rca.55-2.8

Open Access: www.revistas.una.ac.cr/ambientales e-mail: revista.ambientales@una.ac.cr

Pinos-Morocho D., Morales-Matute O. y Durán-López M. E.

y $58 \%$, respectivamente), seguida por Virgenpamba, en donde estas dos categorías alcanzaron el mismo porcentaje (56\%). Además, en esta última localidad, la noción de los SER fue la más baja. Sorprendentemente, la comuna de Zhindilig demostró tener un reconocimiento ligeramente más elevado de los SER y el menor de los SES y SEA. En cuanto a los SES, Aguilán y Virgenpamba también manifestaron una percepción poco significativa.

En la Figura 5 se aprecia que la diferencia de las percepciones entre mujeres y hombres en las 3 comunas no es muy marcada. Los SEA son los servicios más percibidos por el sexo

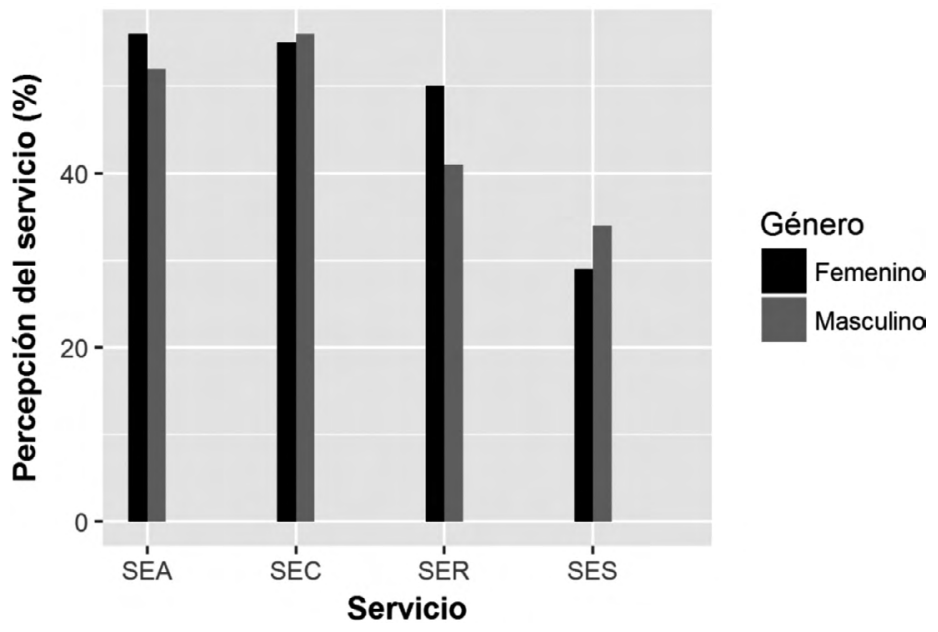

Figura 5. Percepción de servicios ecosistémicos por género, donde SEA: aprovisionamiento, SER: regulación, SEC: culturales, S: soporte.

Figure 5. Perception of ecosystem services per gender. SEA: provisioning, SER: regulation, SEC: cultural, SES: support. femenino sin una diferencia significativa con la apreciación de los hombres por esta misma categoría. Con respecto a los SER, esta fue la categoría que mayor diferencia entre sexos presentó: las mujeres demostraron una percepción más elevada (50 \%) que el sexo masculino (40\% aprox.). En cuanto a los SEC no existe diferencia entre ambos sexos, pero nuevamente puede observarse que los SES son aquellos con los porcentajes más bajos de percepción (mujeres $29 \%$ y hombres $34 \%$ ).

\subsection{Resultados de los análisis de carbono orgánico y densidad aparente}

De todas las muestras analizadas (Cuadro 7), el valor más alto de carbono orgánico fue de $11.92 \%$, que correspondió al Punto 5 (páramo natural), en pendiente de tipo Media a Fuerte ( 25 a $40 \%$ ); mientras que el valor más bajo fue de $7.69 \%$ en el Punto 4 (páramo intervenido), en pendiente de tipo Media (12 a $25 \%$ ). En general, los valores del contenido de carbono no variaron significativamente entre sí, pues las cifras se encuentran en un rango entre aproximadamente 8 y $12 \%$. Los valores de densidad aparente fueron registrados entre $0.33 \mathrm{~g} / \mathrm{cm}^{3}$ (Punto 2: natural, en pendiente Muy suave: 5 a $12 \%$ ) y $0.81 \mathrm{~g} / \mathrm{cm}^{3}$ (Punto 4: intervenido, en pendiente Media: 12 a $25 \%)$.

\begin{tabular}{|c|c|c|c|c|c|}
\hline 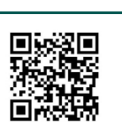 & (c) (1) () () () & 8 & $\frac{2 \%}{\frac{O O}{20}}$ & 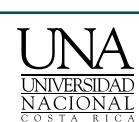 & 169 \\
\hline
\end{tabular}




\section{Revista de CIENCIAS AMBIENTALES Tropical Journal of Environmental Sciences}

Revista de Ciencias Ambientales (Trop J Environ Sci) e-ISSN: 2215-3896

(Julio-Diciembre, 2021) . Vol 55(2): 157-179

DOI: https://doi.org/10.15359/rca.55-2.8

Open Access: www.revistas.una.ac.cr/ambientales e-mail: revista.ambientales@una.ac.cr

Pinos-Morocho D., Morales-Matute O. y Durán-López M. E.

Cuadro 7. Valores de carbono orgánico y densidad aparente del suelo.

Table 7. Organic carbon values and bulk density of the soil.

\begin{tabular}{lcc}
\hline Muestras con relación a la pendiente [\%] & Porcentaje de carbono orgánico [\% C] & Densidad aparente [g/cm $\left.\mathbf{c m}^{3}\right]$ \\
\hline Punto $1(0-2)^{* *}$ & 7.77 & 0.44 \\
Punto $2(2-5)^{*}$ & 10.12 & 0.33 \\
Punto $3(5-12)^{* *}$ & 8.22 & 0.68 \\
Punto $4(12-25)^{* *}$ & 7.69 & 0.81 \\
Punto $5(25-40)^{*}$ & 11.92 & 0.6 \\
Punto $6(40-70)^{*}$ & 10.39 & 0.7 \\
Punto $7(>70)^{*}$ & 10.42 & 0.38 \\
Promedio & 9.5 & 0.56 \\
\hline
\end{tabular}

* Muestreo en sitios naturales.

${ }^{* *}$ Muestreo en sitios intervenidos.

Los valores indicados en el Cuadro 8 se emplearon para estimar el contenido de carbono utilizando la Ecuación 1. La cantidad de carbono existente en los suelos del páramo estudiado, para sitios naturales e intervenidos en conjunto, fue de 392.30 ton $\mathrm{CO}_{2} /$ ha.

Cuadro 8. Valores de cada factor considerado para el cálculo del contenido de carbono.

Table 8. Values of each factor considered to compute the carbon content.

\begin{tabular}{|c|c|}
\hline Factores considerados & Valores \\
\hline Densidad aparente $[\mathrm{g} / \mathrm{cm} 3]$ & 0.56 \\
\hline Profundidad $[\mathrm{cm}]$ & 20 \\
\hline Porcentaje de carbono orgánico - Promedio [\%C] & 9.5 \\
\hline Factor de compensación ${ }^{*}$ & 100 \\
\hline Relación estequiométrica ${ }^{\star *}$ & $44 / 12$ \\
\hline Contenido de carbono en el suelo [ton $\mathrm{CO}_{2} / \mathrm{ha}$ ] & 392.3 \\
\hline
\end{tabular}

* El factor 100 es el resultante de la compensación de las unidades de cm, m y ha.

${ }^{* *}$ La relación $44 / 12$ proviene de la relación estequiométrica de pesos moleculares con la finalidad de conocer cuánto $\mathrm{CO}_{2}$ puede originarse por la descomposición del carbono orgánico.

De acuerdo con el contenido de carbono de los 7 puntos muestreados se plantearon dos escenarios: a) al usar los cálculos de los sitios naturales para estimar el contenido de carbono en el suelo, se obtienen 394.76 ton $\mathrm{CO}_{2} /$ ha. y b) al usar los cálculos de los sitios intervenidos, el contenido de carbono baja a 372.39 ton $\mathrm{CO}_{2} /$ ha. Por consiguiente, en el páramo ubicado al norte del Bosque Protector Cubilán los suelos intervenidos del poseen un $26 \%$ menos en su contenido de carbono en comparación con las muestras de sitios naturales (Cuadro 9).

\begin{tabular}{|c|c|c|c|c|c|}
\hline 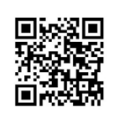 & (c) (1) (9) & $\underset{\text { AMBENTALES }}{\leftrightarrow}$ & $\frac{9 \%}{2 \%}$ & 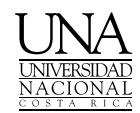 & 170 \\
\hline
\end{tabular}




\section{Revista de CIENCIAS AMBIENTALES Tropical Journal of Environmental Sciences}

Revista de Ciencias Ambientales (Trop J Environ Sci) e-ISSN: 2215-3896

(Julio-Diciembre, 2021) . Vol 55(2): 157-179

DOI: https://doi.org/10.15359/rca.55-2.8

Open Access: www.revistas.una.ac.cr/ambientales e-mail: revista.ambientales@una.ac.cr

Pinos-Morocho D., Morales-Matute O. y Durán-López M. E.

Cuadro 9. Valores considerados en el cálculo del contenido de carbono para los escenarios planteados.

Table 9. Values considered to compute the carbon content for the proposed scenarios.

\begin{tabular}{lcc}
\hline \multicolumn{1}{c}{ Factores considerados } & a. Valores de páramo natural & b. Valores de páramo intervenido \\
\hline Densidad aparente - Promedio $[\mathrm{g} / \mathrm{cm} 3]$ & 0.5 & 0.64 \\
Profundidad [cm] & 20 & 20 \\
Porcentaje de carbono orgánico - Promedio [\% C] & 10.71 & 7.89 \\
Factor de compensación & 100 & 100 \\
Relación estequiométrica & $44 / 12$ & $44 / 12$ \\
Contenido de carbono en el suelo [ton $\mathrm{CO}_{2} / \mathrm{ha}$ ] & $\mathbf{3 9 4 . 7 6}$ & $\mathbf{3 7 2 . 3 9}$ \\
\hline
\end{tabular}

\subsection{Valor económico total del contenido de} carbono en el suelo del páramo

El valor económico total más alto corresponde al que se proyectó para el año 2030 y el más bajo corresponde al año 2017, debido a la disminución del precio que sufrió la tonelada de $\mathrm{CO}_{2}$ en ese período. Con las cifras de la Figura 6 se evidencia que el precio del carbono disminuyó entre 2011 y 2017. Sin embargo, si se mantiene el contenido de carbono en el suelo, proyectado con los cálculos de este estudio, se esperaría que el valor económico incremente notablemente para los años 2020 y 2030 .

Si traducimos a términos económicos la capacidad que tienen los suelos de páramos naturales e intervenidos para contener carbono, y los comparamos entre sí, el valor monetario del páramo intervenido pierde un $6 \%$ (1.7 millones de USD) con respecto al valor del páramo natural. Además, en la Figura 7 se compara el estado del páramo en el año 2018 (actual) y las dos condiciones consideradas (páramo natural e intervenido) en términos de valor económico para los años analizados. El valor de carbono para el estado actual resulta de un promedio obtenido de los 7 sitios considerados.

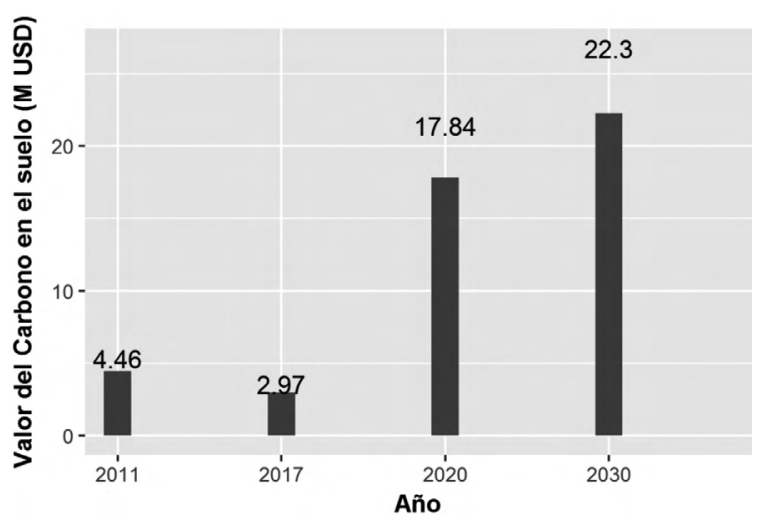

Figura 6. Proyección del valor económico del carbono a través del tiempo.

Figure 6. Projection of the economic value of carbon over time.

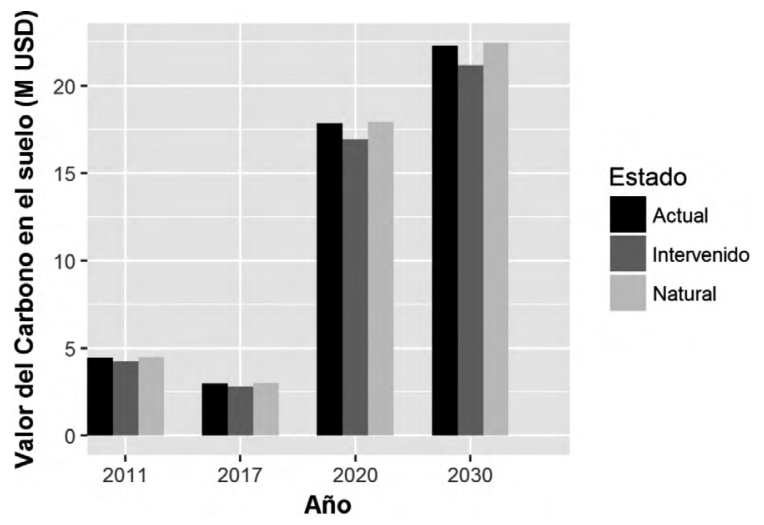

Figura 7. Valor económico del carbono en función de los escenarios planteados.

Figure 7. Economic value of carbon based on the proposed scenarios.

\begin{tabular}{|c|c|c|}
\hline 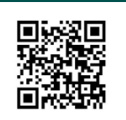 & (c) (i) () () & 171 \\
\hline
\end{tabular}




\section{Revista de CIENCIAS AMBIENTALES Tropical Journal of Environmental Sciences}

Revista de Ciencias Ambientales (Trop J Environ Sci)

e-ISSN: 2215-3896

(Julio-Diciembre, 2021) . Vol 55(2): 157-179

DOI: https://doi.org/10.15359/rca.55-2.8

Open Access: www.revistas.una.ac.cr/ambientales e-mail: revista.ambientales@una.ac.cr

Pinos-Morocho D., Morales-Matute O. y Durán-López M. E.

\section{Discusión}

\subsection{Importancia del estudio de la percepción ambiental para la conservación}

Los habitantes (mujeres y hombres) de las comunas aledañas al páramo perciben las cuatro categorías de servicios ecosistémicos y reconocen que estos contribuyen a mejorar su calidad de vida. Estos resultados coinciden con lo reportado en otros trabajos (Espinosa et al., 2014; Hofstede et al., 2014), donde el páramo es visualizado desde la sabiduría popular como un ecosistema de características biológicas y culturales especiales y únicas.

No obstante, se evidenció una jerarquización de las categorías de servicios ecosistémicos percibidas, pero sin que exista una diferencia marcada entre el sexo femenino y masculino. Puesto que la percepción varía y depende del acceso que las personas tengan a los recursos (Díaz et al., 2011), al tratarse de comunas rurales, su interacción con los recursos naturales es mayor.

Los servicios ecosistémicos de abastecimiento (SEA) fueron los más reconocidos, dado que en economías de subsistencia estos servicios son los más apreciados (Caballero et al., 2017). En esta investigación, como en la de Farley \& Bremer, (2017) e Higuera et al., (2013) el principal elemento que los comuneros perciben recibir del páramo es el agua, y reconocen a este ecosistema como un elemento indispensable para su vida. De igual manera los servicios ecosistémicos culturales (SEC) registraron un alto nivel de percepción en el entorno agropecuario del estudio, contradiciendo a lo reportado por Martín-López et al., (2012) quienes indican que esta categorización es más valorada en entornos industrializados. La considerable percepción de los SEC indica que los pobladores (mujeres y hombres) identifican al páramo como un componente relevante para su historia y cultura colectiva.

Por otro lado, los servicios ecosistémicos de soporte (SES) fueron los menos reconocidos. Como era de esperarse, no es sencillo distinguir esta categoría porque involucra una mayor comprensión de los procesos y componentes del ecosistema (Camacho \& Ruiz, 2012).

Si bien el páramo es reconocido y percibido como un sólido proveedor de beneficios a nivel global, en las últimas décadas se ha evidenciado su deterioro en la región andina. En consecuencia, los esfuerzos por conservar este ecosistema deben incluir las percepciones ambientales y las actividades de sus habitantes, para involucrarlos en la búsqueda y aplicación de mejores estrategias de manejo (Espinosa et al., 2014; Tapia et al., 2017).

\subsection{Contenido de carbono en los suelos de páramo: Una de las mayores reservas de carbono}

Se determinó que el contenido de carbono orgánico de los suelos del páramo en estudio es alto, pues los resultados sobrepasaron el valor referencial de $5 \%$ que se establece en otros trabajos realizados en el mismo ecosistema en Sudamérica y México (Gutiérrez, 2015; Vela et al., 2012).

Si comparamos el contenido de carbono de los suelos de páramo con la vegetación de un bosque tropical, este almacena hasta 250 toneladas de carbono elemental (una unidad de carbono elemental equivale a 3.6 unidades de $\mathrm{CO}_{2}$ ) por ha, lo que equivale a 900 toneladas de $\mathrm{CO}_{2} \mathrm{y}$,

\begin{tabular}{|c|c|c|c|c|c|}
\hline 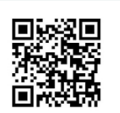 & (c) (1) (9) & 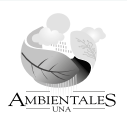 & $\frac{O \%}{2)}$ & 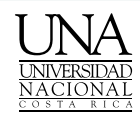 & 172 \\
\hline
\end{tabular}




\section{Revista de CIENCIAS AMBIENTALES Tropical Journal of Environmental Sciences}

Revista de Ciencias Ambientales (Trop J Environ Sci)

e-ISSN: 2215-3896

(Julio-Diciembre, 2021) . Vol 55(2): 157-179

DOI: https://doi.org/10.15359/rca.55-2.8

Open Access: www.revistas.una.ac.cr/ambientales e-mail: revista.ambientales@una.ac.cr

Pinos-Morocho D., Morales-Matute O. y Durán-López M. E.

en el páramo la vegetación tiene 20 toneladas de carbono elemental equivalente a 72 toneladas de $\mathrm{CO}_{2}$ (Gutiérrez, 2015). Sin embargo, con respecto a la cantidad de carbono secuestrada en el suelo, las cifras muestran que en un bosque tropical el suelo contiene 50 toneladas de carbono elemental (en suelos con densidad aparente de $1 \mathrm{~kg} / \mathrm{l}$ ), mientras que en el suelo de páramo se ha registrado hasta 1700 toneladas de carbono por ha (en suelos con densidad aparente de $0.5 \mathrm{~kg} / \mathrm{l}$ ) (Gutiérrez, 2015). De manera que los suelos de estos ecosistemas de montaña constituyen una de las mayores reservas de carbono en el mundo, gracias a las bajas temperaturas que minimizan las tasas de descomposición de materia orgánica. Por lo tanto, conservar el páramo es tan importante como conservar los bosques tropicales.

La diferencia entre los resultados de las muestras tomadas en sitios naturales e intervenidos indica que, en el primer escenario, el contenido de carbono fue mayor (10.71\%) que en el segundo (7.89 \%). Esto concuerda con el estudio de Castro (2011), realizado en el Frente Suroccidental de Tungurahua (Ecuador), donde el promedio de carbono en el suelo del páramo natural fue de $13.50 \%$; mientras que en el páramo intervenido se encontró $3.50 \%$. Los datos de ambos estudios evidencian que hay una mayor pérdida del contenido de carbono en los suelos del páramo, cuando la superficie es sometida a varios procesos antrópicos (agricultura, ganadería, infraestructura, entre otros).

Los porcentajes de almacenamiento de carbono reportados para el páramo intervenido en este trabajo son mayores a los obtenidos por Castro (2011). Esto puede deberse a que el páramo estudiado entró en un proceso de conservación desde 2006, al suspender las actividades de agricultura y ganadería (Fundación ECOHOMODE, 2006), en contraste con la intervención alta que presentaba el páramo del Frente Suroccidental de Tungurahua. Adicionalmente, dada la alta capacidad de retención de humedad que presentan los suelos de páramo, sumada a las condiciones de clima, en donde la precipitación está presente durante largos periodos en el año, se posibilita la recuperación de la vegetación en la zona (Íñiguez et al., 2016).

Adicionalmente, varios estudios realizados en páramos de la sierra centro-sur ecuatoriana en Loja, Tungurahua y Chimborazo (Castro, 2011; Chamorro et al., 2017; Convención de Ramsar y Grupo de Contacto EHAA, 2008; Ministerio del Ambiente de Ecuador, 2015) evidencian que, ante una continua intervención antropogénica (agricultura y ganadería), existe una pérdida potencial en el contenido de carbono orgánico y su valor económico. También afirman que un páramo conservado puede proveer de mejor manera los servicios ecosistémicos y a las poblaciones que dependen directamente de él.

\subsection{Importancia de la valoración económica}

En otras investigaciones (Castañeda \& Montes, 2017; Cunalata et al., 2013) se ha podido constatar que el aumento de emisiones de $\mathrm{CO}_{2}$ puede agravarse por la degradación de suelos de páramo, pues al deteriorarse, el carbono almacenado podría descomponerse y liberarse. Ante tal premisa, este estudio demuestra que el valor económico de la captura de carbono facilita definir estrategias conservacionistas, por representar una buena opción de mitigación (Camacho

\begin{tabular}{|c|c|c|c|c|c|}
\hline 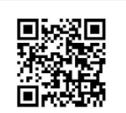 & (c) (i) (5) & 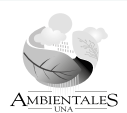 & $\frac{10 \%}{\frac{9}{2}}$ & $\frac{\text { UNA }}{\frac{\text { UNIVERSIDAD }}{\text { UNACIONAL }}}$ & 173 \\
\hline
\end{tabular}




\section{Revista de CIENCIAS AMBIENTALES Tropical Journal of Environmental Sciences}

Revista de Ciencias Ambientales (Trop J Environ Sci)

e-ISSN: 2215-3896

(Julio-Diciembre, 2021) . Vol 55(2): 157-179

DOI: https://doi.org/10.15359/rca.55-2.8

Open Access: www.revistas.una.ac.cr/ambientales e-mail: revista.ambientales@una.ac.cr

Pinos-Morocho D., Morales-Matute O. y Durán-López M. E.

\& Ruiz, 2012). Según el quinto informe del Panel Intergubernamental del Cambio Climático (IPCC, 2014), la valoración económica del almacenamiento de carbono es una de las opciones más costo-efectivas a aplicarse para conservar reservorios de carbono existentes en los suelos.

De acuerdo con los precios del carbono para los años 2017 (cifra más baja) y 2030 (cifra más alta), la superficie total del páramo en estudio representaría 2.97 y 29.73 millones de dólares americanos, respectivamente, al capturar cantidades considerables de carbono en sus suelos. Por ello, este páramo se postula como una zona importante para ingresar a la categoría de Áreas Protegidas, porque además de conservarse debería recuperar sus sitios intervenidos para incrementar la capacidad de mitigación. Estudios sobre la importancia de los suelos de este ecosistema indican que páramos alterados pueden recuperarse mediante programas de protección, específicamente para restablecer sus propiedades hidrofísicas, el contenido de materia orgánica (carbono orgánico), y el agua disponible (Bernal, 2017; Cárdenas \& Tobón, 2017).

\section{Conclusiones}

El cambio climático representa un reto considerable para las poblaciones humanas; por ello, incorporar la percepción ambiental en los planes de manejo de los recursos naturales es algo imprescindible. Este estudio demostró que los habitantes de las 3 comunas perciben las 4 categorías de servicios ecosistémicos del páramo aledaño en el siguiente orden: SEA-SEC, SER, SES. También se evidenció que el sexo no es un factor determinante para una elevada percepción de los SES y SEA, lo que confirma el gran sentido de pertenencia y aprecio que los comuneros tienen hacia el páramo.

De acuerdo con los escenarios planteados en este estudio, los suelos intervenidos del páramo poseen un $26 \%$ menos en el contenido de carbono, en comparación con el carbono en suelos naturales. Si traducimos esta capacidad a términos monetarios, y los contrastamos entre sí, el monto económico del páramo intervenido pierde un 6 \% (1.7 millones de USD) del valor con respecto al monto proporcionado por el páramo natural. Por consiguiente, al conservar el suelo del páramo, se disminuye la cantidad de $\mathrm{CO}_{2}$ liberada a la atmósfera, evidenciando el servicio ecosistémico de captura de carbono, que se incluye como una opción de mitigación de los efectos del cambio climático. El contenido de carbono por hectárea en suelos de páramo es mayor que el que contiene la vegetación de una selva tropical, lo que lo convierte en uno de los mayores reservorios de carbono.

Al conservar el páramo ubicado al norte del Bosque Protector Cubilán se asegura una cantidad importante de contenido de carbono (392.30 ton $\mathrm{CO}_{2} /$ ha), que en el mercado de carbono representa un costo monetario de 23.78 millones de USD para el año 2020 y de 29.73 millones de USD para el año 2030, considerando el objetivo de temperatura establecido en el Acuerdo de París.

\begin{tabular}{|c|c|c|c|c|c|}
\hline 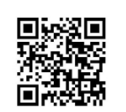 & (c) (i) $(9)$ & $\theta_{\text {AMBIENTALS }}$ & $\frac{O \%}{20}$ & $\frac{\text { UNA }}{\frac{\text { UNIVERSIDAD }}{\text { NACIONAL }}}$ & 174 \\
\hline
\end{tabular}




\section{Revista de CIENCIAS AMBIENTALES Tropical Journal of Environmental Sciences}

Revista de Ciencias Ambientales (Trop J Environ Sci) e-ISSN: 2215-3896

(Julio-Diciembre, 2021) . Vol 55(2): 157-179 DOI: https://doi.org/10.15359/rca.55-2.8 Open Access: www.revistas.una.ac.cr/ambientales e-mail: revista.ambientales@una.ac.cr

Pinos-Morocho D., Morales-Matute O. y Durán-López M. E.

\section{7. Ética y conflicto de intereses}

Las personas autoras declaran que han cumplido totalmente con todos los requisitos éticos y legales pertinentes, tanto durante el estudio como en la producción del manuscrito; que no hay conflictos de intereses de ningún tipo; que todas las fuentes financieras se mencionan completa y claramente en la sección de agradecimientos; y que están totalmente de acuerdo con la versión final editada del artículo.

\section{Agradecimientos}

Agradecemos al Municipio de Azogues, por facilitar el ingreso al sitio de estudio, propiciar el acercamiento a las comunas y proporcionar la cartografía base. También agradecemos a los presidentes de cada comuna y a los comuneros que participaron en este estudio. De manera especial, agradecemos al Programa para el Manejo del Agua y del Suelo (PROMAS), por proporcionar los instrumentos para la realización del muestreo, así como el acceso al laboratorio para analizar las muestras. Al Dr. Rodrigo Caroca Cáceres por su colaboración en la revisión del escrito (textos traducidos al idioma inglés). Finalmente, agradecemos a la Revista y las personas revisoras anónimas por sus oportunas observaciones en la versión final del documento.

\section{Referencias}

Ayala, L., Villa, M., Aguirre, Z., Aguirre, N. (2014). Cuantificación del carbono en los páramos del parque nacional Yacuri, provincias de Loja y Zamora Chinchipe, Ecuador. CEDAMAZ, 4(1), 45-52.

Barreno, M. A. (2013). Obtención de azúcares reductores por hidrólisis ácida a partir de dos variedades de paja (Calamagrostis intermedia y Stipa ichu) [Tesis de pregrado]. Universidad Central del Ecuador.

Bernal, I. M. (2017). Conflictos socioambientales en el páramo de Guacheneque y estrategias de conservación para el ordenamiento ambiental regional [Tesis de maestría]. Universidad Nacional de Colombia.

Caballero, V., Alday, J. G., Amigo, J., Caballero, D., Carrasco, J. C., McLaren, B., Onaindia, M. (2017). Social Perceptions of Biodiversity and Ecosystem Services. Ecología humana, 45(1), 475-486. https://doi.org/10.1007/s10745-017-9921-6

Calderón, L., Hernández, A., López, J. L. (2013). Valoración de los bienes y servicios ambientales provistos por el Páramo de Santurbán (p. 81). FEDESARROLLO, Centro de Investigación Económica y Social. Bogotá.

Camacho, M. (2013). Los páramos ecuatorianos: Caracterización y consideraciones para su conservación y aprovechamiento sostenible. ANALES de la Universidad Central del Ecuador, 77-92. https://doi.org/10.29166/anales.v1i372.1241

\begin{tabular}{|c|c|c|c|c|c|}
\hline 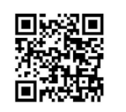 & (c) $\underset{\mathrm{BY}}{(1)}(0)$ & $\underset{\text { AMBENETILES }}{3}$ & $\frac{1 \%}{2 \%}$ & $\frac{\text { UNA }}{\frac{\text { UNIVERSIDAD }}{\text { NACIONAL }}}$ & 175 \\
\hline
\end{tabular}




\section{Revista de CIENCIAS AMBIENTALES Tropical Journal of Environmental Sciences}

Revista de Ciencias Ambientales (Trop J Environ Sci) e-ISSN: 2215-3896

(Julio-Diciembre, 2021) . Vol 55(2): 157-179 DOI: https://doi.org/10.15359/rca.55-2.8 Open Access: www.revistas.una.ac.cr/ambientales e-mail: revista.ambientales@una.ac.cr

Pinos-Morocho D., Morales-Matute O. y Durán-López M. E.

Camacho, V., Ruiz, A. (2012). Marco conceptual y clasificación de los servicios ecosistémicos. Bio Ciencias, 1(4), 3-15. https://doi.org/10.15741/rev\%20bio\%20ciencias.v1i4.19

Cárdenas, M. F., Tobón, C. (2017). Recuperación del funcionamiento hidrológico de ecosistemas de páramo en Colombia. Revista U.D.C.A Actualidad \& Divulgación Científica, 20(2), 403-412. https://doi.org/10.31910/rudca.v20.n2.2017.381

Castañeda, A. E. (2016). Carbono almacenado en biomasa aérea y suelo en el ecosistema de páramo (p. 61). Universidad Nacional Abierta y a Distancia-UNAD. Colombia. https://doi. org/10.18041/entramado.2017v13n1.25112

Castañeda, A. E., Montes, C. R. (2017). Carbono almacenado en páramo andino. Entramado, 13(1), 210-221. http://dx.doi.org/10.18041/entramado.2017v13n1.25112

Castro, M. (2011). Una valoración económica del almacenamiento de agua y carbono en los bofedales de los páramos ecuatorianos - La experiencia en Oña-Nabón-Saraguro-Yacuambi y el Frente Suroccidental de Tungurahua. EcoCiencia/Wetlands International/UTPL/ MAE. Quito. https://ecociencia.org/una-valoracion-economica-del-almacenamiento-de-agua-y-carbono-en-los-bofedales-de-los-paramos-ecuatorianos/

Chamorro, S., Villarroel, M., González, M. A., Palacios, T. (2017). Almacenamiento de agua y fijación de carbono en Reserva Ecológica El Ángel y su impacto en el uso del suelo. FIGEMPA: Investigación y Desarrollo, 1(2), 42-48. https://doi.org/10.29166/revfig.v1i1.54

Convención de Ramsar y Grupo de Contacto EHAA. (2008). Estrategia regional de conservación y uso sostenible de los humedales altoandinos. Agua, vida, futuro (p. 54). Gobiernos de Ecuador y Chile, CONDESAN y TNC-Chile.

Córdova, M., Célleri, R., Shellito, C. J., Orellana, J., Abril, A., Carrillo, G. (2016). Near-Surface Air Temperature Lapse Rate Over Complex Terrain in the Southern Ecuadorian Andes: Implications for Temperature Mapping. Arctic, Antarctic, and Alpine Research, 48(4), 673-684. http://dx.doi.org/10.1657/AAAR0015-077

Crespo, P., Célleri, R., Buytaert, W., Feyen, J., Iñiguez, V., Borja, P., De Bievre, B. (2009). Land use change impacts on the hydrology of wet Andean páramo ecosystems. Status and Perspectives of Hydrology in Small Basins, 71-76. https://doi.org/10.13140/2.1.5137.6320

Cunalata, C., Inga, C., Álvarez, G., Recalde, C., Echeverría, M. (2013). Determinación de carbono orgánico total presente en el suelo y la biomasa de los páramos de las comunidades de Chimborazo y Shobol Ilinllin en Ecuador. Boletín del Grupo Español Carbón, 27, 10-13.

Díaz, S., Quétier, F., Cáceres, D. M., Trainor, S. F., Pérez, N., Bret, M. S., Finegan, B., Peña, M., Poorter, L. (2011). Linking functional diversity and social actor strategies in a framework for interdisciplinary analysis of nature's benefits to society. Proceedings of the National

\begin{tabular}{|c|c|c|c|c|}
\hline 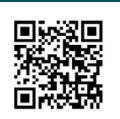 & (c) (1) () () & $\Leftrightarrow$ & $\frac{10 \%}{\text { euna }}$ & 176 \\
\hline
\end{tabular}




\section{Revista de CIENCIAS AMBIENTALES Tropical Journal of Environmental Sciences}

Revista de Ciencias Ambientales (Trop J Environ Sci) e-ISSN: 2215-3896

(Julio-Diciembre, 2021) . Vol 55(2): 157-179 DOI: https://doi.org/10.15359/rca.55-2.8 Open Access: www.revistas.una.ac.cr/ambientales e-mail: revista.ambientales@una.ac.cr

Pinos-Morocho D., Morales-Matute O. y Durán-López M. E.

Academy of Sciences of the United States of America, 108(3), 895-902. https://doi.org/10.1073/ pnas. 1017993108

Durán, M. E. (2013). Percepciones sobre la Naturaleza y actitudes ambientales de los niños y niñas en escuelas urbanas de cuatro cantones de la provincia de Heredia, Costa Rica [Tesis de maestría]. Universidad de Costa Rica.

Durán, M. E., Barrientos, Z., Charpentier, C. (2016). Percepción ambiental de escolares urbanos: Influencia de áreas verdes, financiamiento y sexo en Costa Rica. Cuadernos de Investigación UNED, 8(1), 31-39. https://doi.org/10.22458/urj.v8i1.1220

Durand, L. (2008). De las percepciones a las perspectivas ambientales. Una reflexión teórica sobre la antropología y la temática ambiental. Nueva Antropología, 21(68), 75-87.

Espinosa, N., Chaparro, J. A., Chaparro, N. Y. (2014). Conocimiento local, sobre uso y manejo de recursos naturales del Páramo el Consuelo. Cultura Científica, 46-55.

FAO. (2002). Captura de carbono en suelos para un mejor manejo de la tierra. Organización de las Naciones Unidas para la Agricultura y la Alimentación. Informes sobre recursos mundiales de suelos \#96. http://www.fao.org/3/bl001s/bl001s.pdf

Farley, K. A., Anderson, W., Bremer, L. L., Harden, C. P. (2011). Compensation for ecosystem services: An evaluation of efforts to achieve conservation and development in Ecuadorian páramo grasslands. Environmental Conservation, 38(4), 393-405. https://doi.org/doi:10.1017/ S037689291100049X

Farley, K. A., Bremer, L. L. (2017). "Water Is Life”: Local Perceptions of Páramo Grasslands and Land Management Strategies Associated with Payment for Ecosystem Services. Annals of the American Association of Geographers, 1(1), 1-11. http://dx.doi.org/10.1080/2 4694452.2016.1254020

Fernández, Y. (2008). ¿Por qué estudiar las percepciones ambientales? Una revisión de la literatura mexicana con énfasis en Áreas Naturales Protegidas. Espiral, Estudios sobre Estado y Sociedad, 15(43), 179-202.

Fundación ECOHOMODE. (2006). Plan de manejo participativo de los recursos naturales del Bosque Protector Cubilán (p. 92).

Guamán, C. A. (2018). Validación del método para determinar materia orgánica en el suelo por la técnica de Walkley And Black [Tesis de pregrado]. Universidad de Cuenca. http://dspace. ucuenca.edu.ec/handle/123456789/31352

Gutiérrez, M. L. (2015). Carbono como indicador de degradación de la calidad del suelo bajo diferentes coberturas en el páramo de Guerrero [Tesis de maestría]. Universidad Nacional de Colombia.

\begin{tabular}{|c|c|c|c|c|c|}
\hline 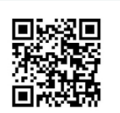 & (c) (1) (9) & 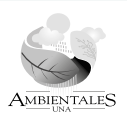 & $\frac{O \%}{2)}$ & 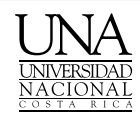 & 177 \\
\hline
\end{tabular}




\section{Revista de CIENCIAS AMBIENTALES Tropical Journal of Environmental Sciences}

Revista de Ciencias Ambientales (Trop J Environ Sci) e-ISSN: 2215-3896

(Julio-Diciembre, 2021). Vol 55(2): 157-179 DOI: https://doi.org/10.15359/rca.55-2.8 Open Access: www.revistas.una.ac.cr/ambientales e-mail: revista.ambientales@una.ac.cr

Pinos-Morocho D., Morales-Matute O. y Durán-López M. E.

Hayes, T., Murtinho, F., Wolff, H. (2015). An institutional analysis of Payment for Environmental Services on collectively managed lands in Ecuador. Ecological Economics, 118, 81-89. http://dx.doi.org/10.1016/j.ecolecon.2015.07.017

Hayes, T., Murtinho, F., Wolff, H. (2017). The Impact of Payments for Environmental Services on Communal Lands: An Analysis of the Factors Driving Household Land-Use Behavior in Ecuador. World Development, 1-20. http://dx.doi.org/10.1016/j.worlddev.2017.01.003

Higuera, D., Martín, B., Sánchez, A. (2013). Social preferences towards ecosystem services provided by cloud forests in the neotropics: Implications for conservation strategies. Regional Environmental Change, 13(1), 861-872. https://doi.org/DOI 10.1007/s10113-012-0379-1

Hofstede, R., Calles, J., López, V., Polanco, R., Torres, F., Ulloa, J., Vásquez, A., Cerra, M. (2014). Los páramos andinos: ¿Qué sabemos? Estado de conocimiento sobre el impacto del cambio climático en el ecosistema páramo (p. 79). UICN.

Instituto Nacional Autónomo de Investigaciones Agropecuarias (2016). Muestreo de suelos para el análisis químico (p. 6). INIAP. Ecuador.

Íñiguez, V., Morales, O., Cisneros, F., Bauwens, W., Wyseure, G. (2016). Analysis of the drought recovery of Andosols on southern Ecuadorian Andean páramos. Hydrology and Earth System Sciences, 20(6), 2421-2435. https://doi.org/10.5194/hess-20-2421-2016

Intergovernmental Panel on Climate Change (2014). Cambio climático 2014: Informe de síntesis (p. 157). IPCC. Ginebra, Suiza.

MAGAP, Programa de Regulación y Administración de Tierras Rurales, EC (PRAT), Instituto Espacial Ecuatoriano (IEE) (2010). Anexo No. 51-C. Metodología del mapa de aptitudes agrícolas para el Ecuador Continental (p. 16). http://balcon.magap.gob.ec/mag01/magapaldia/ Anexos/3.4\%20Anexo\%2051\%20C\%20METODOLOGI\%CC\%81A\%20PARA\%20EL\%20 MAPA\%20DE\%20APTITUDES\%20AGRI\%CC\%81COL.pdf

Martín-López, B., Iniesta, I., García, M., Palomo, I., Casado, I., García Del Amo, D., Gómez, E., Oteros, E., Palacios, I., Willaarts, B., González, J. A., Santos, F., Onaindia, M., López, C., Montes, C. (2012). Uncovering ecosystem service bundles through social preferences. PLoS One, 7(6), 1-11. https://doi.org/10.1371/journal.pone.0038970. PMid:22720006 PMCid:PMC3377692

Mena, P., Medina, G. (2002). La biodiversidad de los páramos en el Ecuador. En: Mena, P., Medina, G. y Hofstede, R. (eds.). Los páramos del Ecuador: Particularidades, problemas y perspectivas (p. 26). Abya Yala/Proyecto Páramo. Quito.

Millennium Ecosystem Assessment (2005). Ecosystems and Human Well-Being: Synthesis. Island Press. Washington D.C. https://www.millenniumassessment.org/documents/document.356.aspx.pdf

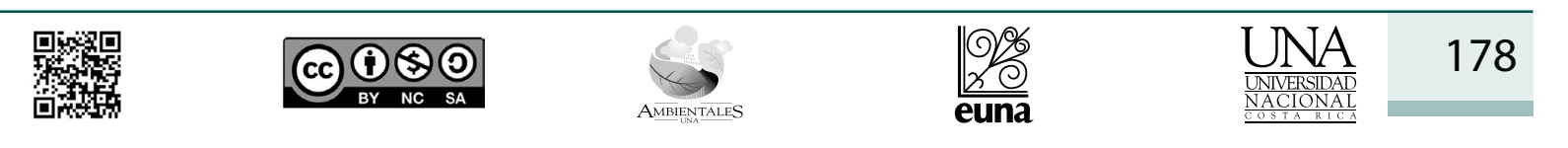




\section{Revista de CIENCIAS AMBIENTALES Tropical Journal of Environmental Sciences}

Revista de Ciencias Ambientales (Trop J Environ Sci) e-ISSN: 2215-3896

(Julio-Diciembre, 2021) . Vol 55(2): 157-179 DOI: https://doi.org/10.15359/rca.55-2.8 Open Access: www.revistas.una.ac.cr/ambientales e-mail: revista.ambientales@una.ac.cr

Pinos-Morocho D., Morales-Matute O. y Durán-López M. E.

Ministerio del Ambiente de Ecuador (2015). Humedales del Ecuador. Ministerio del Ambiente. http://suia.ambiente.gob.ec/web/humedales/documentos

Moreano, M. (2012). Socio bosque y el capitalismo verde. Pensando la coyuntura. Los cuadernos de La línea de fuego, 113-137. Ediciones Abya-Yala y Fundación Rosa Luxemburg. Quito.

Otzen, T., Manterola, C. (2017). Técnicas de muestreo sobre una población a estudio. International Journal of Morphology, 35(1), 227-232. http://dx.doi.org/10.4067/S0717-95022017000100037

Serrano, D., Galárraga, R. (2015). El páramo andino: Características territoriales y estado ambiental. Aportes interdisciplinarios para su conocimiento. Estudios Geográficos, LXXV(I), 369393. https://doi.org/10.3989/estgeogr.201513

Shepardson, D. P., Niyogi, D., Choi, S., Charusombat, U. (2009). Seventh grade students' conceptions of global warming and climate change. Environmental Education Research, 5(15), 549-570. http://dx.doi.org/10.1080/13504620903114592

Swift, R. S. (2001). Sequestration of carbon by soil. Soil Science, 166(11), 858-871. https://doi. org/10.1097/00010694-200111000-00010

Tapia, M. V., Jiménez, A., Cuásquer, E., Cabrera, C. A., Caicedo, E. M. (2017). Evaluación del programa de conservación del ecosistema páramo y sus efectos socioambientales en una comuna rural. CFORES Revista Cubana de Ciencias Forestales, 5(2), 287-300.

Vela, G., López, J., Rodríguez, M. de L. (2012). Niveles de carbono orgánico total en el Suelo de Conservación del Distrito Federal, centro de México. Investigaciones Geográficas, 77, 18-30. https://doi.org/10.14350/rig.31007

Villar, B., Tosquy, O. H., López, E., Esqueda, V. A., Palacios, G. (2013). Impacto de la pendiente y tres sistemas de producción sobre el escurrimiento, la erosión y el rendimiento de maíz. Tropical and Subtropical Agroecosystems, 16(3), 497-504.

World Bank, Ecofys, Vivid Economics (2017). State and Trends of Carbon Pricing 2017 (p. 104). World Bank Group. https://doi.org/10.1596/978-1-4648-1218-7

Yang, F., Zhang, G.-L., Yang, J.-L., Li, D.-C., Zhao, Y.-G., Liu, F., Yang, R.-M., Yang, F. (2014). Organic matter controls of soil water retention in an alpine grassland and its significance for hydrological processes. Journal of Hydrology, 519(D), 3086-3093. http://dx.doi.org/10.1016/j. jhydrol.2014.10.054

\begin{tabular}{|c|c|c|c|c|c|}
\hline 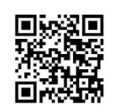 & (c) (1) () (2) & $\underset{\text { AMBENENALIES }}{\infty}$ & $\frac{1 \%}{\text { euna }}$ & 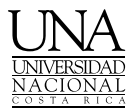 & 179 \\
\hline
\end{tabular}

